\title{
Fatal toxicity of chloroquine or hydroxychloroquine with metformin in mice
}

\author{
N.V Rajeshkumar ${ }^{1}$, Shinichi Yabuuchi ${ }^{2}$, Shweta G. Pai ${ }^{2}$, Anirban Maitra ${ }^{3}$, \\ Manuel Hidalgo ${ }^{4}$ and Chi V. Dang ${ }^{5}$
}

\begin{abstract}
${ }^{1}$ Department of Oncology, Pathology, Radiation Oncology and Molecular Radiation Sciences, Johns Hopkins University School of Medicine, Baltimore, MD.

2Department of Pathology, Johns Hopkins University School of Medicine, Baltimore, MD.

${ }^{3}$ Sheikh Ahmed Center for Pancreatic Cancer Research, M.D. Anderson Cancer Center, Houston, TX.

${ }^{4}$ Division of Hematology and Medical Oncology, Weill Department of Medicine, Sandra and Edward Meyer Cancer Center, Weill Cornell Medicine, New York, NY.

${ }^{5}$ Ludwig Institute for Cancer Research, New York, NY.
\end{abstract}

Corresponding authors: NV Rajeshkumar, Ph.D, Department of Radiation Oncology and Molecular Radiation Sciences, Johns Hopkins University School of Medicine, 1550 Orleans

Street, CRB II, Room 443, Baltimore, MD 21287. Phone: 410-955-7312; Fax: 410-502-2821

E-mail: rnv1@jhmi.edu

Chi V. Dang, MD. Ph.D, Ludwig Institute for Cancer Research, 666 Third Avenue, 28th Floor, New York, NY 10017. Phone: 212-450-1566; Fax: 212- 450-1545; E-mail: cdang@licr.org 


\begin{abstract}
Guided by the principle of primum non nocere (first do no harm), we report a cautionary note on the potential fatal toxicity of chloroquine (CQ) or hydroxychloroquine (HCQ) in combination with anti-diabetic drug metformin. We observed that the combination of CQ or HCQ and metformin, which were used in our studies as potential anti-cancer drugs, killed $30-40 \%$ of mice. While our observations in mice may not translate to toxicity in humans, the reports that CQ or HCQ has anti-COVID-19 activity, the use of CQ resulting in toxicity and at least one death, and the recent Emergency Use Authorization (EUA) for CQ and HCQ by the US Food and Drug Administration (FDA) prompted our report. Here we report the lethality of CQ or HCQ in combination with metformin as a warning of its potential serious clinical toxicity. We hope that our report will be helpful to stimulate pharmacovigilance and monitoring of adverse drug reactions with the use of $\mathrm{CQ}$ or $\mathrm{HCQ}$, particularly in combination with metformin.
\end{abstract}


Introduction. Guided by the principle of primum non nocere (first do no harm), we report a cautionary note on the potential fatal toxicity of chloroquine (CQ) or hydroxychloroquine (HCQ) in combination with anti-diabetic drug metformin. We observed that the combination of CQ or HCQ and metformin, which were used in our studies as potential anti-cancer drugs, killed 30$40 \%$ of mice. While our observations in mice may not translate to toxicity in humans, the reports that CQ or HCQ has anti-COVID-19 activity [1], the use of CQ resulting in toxicity and at least one death, and the recent Emergency Use Authorization (EUA) for CQ and HCQ by the US Food and Drug Administration (FDA) prompted our report. Here we report the lethality of CQ or $\mathrm{HCQ}$ in combination with metformin as a warning of its potential serious clinical toxicity. We hope that our report will be helpful to stimulate pharmacovigilance and monitoring of adverse drug reactions with the use of $\mathrm{CQ}$ or $\mathrm{HCQ}$, particularly with metformin.

Methods. Animal experiments were conducted following approval by the Animal Care and Use Committee guidelines of the Johns Hopkins University (Baltimore, MD). Tumor bearing or nontumor bearing immunocompromised mice were injected with $100 \mu \mathrm{L}$ of saline vehicle, chloroquine (CQ, $60 \mathrm{mg} / \mathrm{kg}$ ), hydroxychloroquine (HCQ, $60 \mathrm{mg} / \mathrm{kg}$ ) and/or metformin (250 $\mathrm{mg} / \mathrm{kg}$ ) once daily intraperitoneally daily for 4 weeks as described [2 3]. A combination of CQ and metformin in the above-mentioned dose and frequency was administered to animals in the combination treatment group. In a separate study, non-tumor bearing immunocompromised and immunocompetent mice were treated with HCQ and metformin once daily in the abovementioned dose for 38 days. Blood was drawn for chemistry and hematology via cardiac puncture, and organs and tissues were harvested, examined and processed for transmission electron microscopy as described [3].

Results. Based on our previous findings that metformin and CQ or HCQ curbed the growth of human pancreatic xenografts in athymic nude mice [2 3], we sought to determine whether the combination of metformin, which inhibits mitochondrial Complex I, and CQ that inhibits autophagy could be synergistic as an anti-cancer metabolic cocktail. In contrast to single agent 
metformin or $\mathrm{CQ}$, which have anti-tumor activity, we found that the combination of metformin and CQ was lethal in $40 \%$ of tumor bearing or non-tumor bearing mice (Figure 1A).

To determine whether HCQ is similarly lethal in combination with metformin, we tested the metformin $+\mathrm{HCQ}$ combination in non-tumor bearing nude mice, which showed a $40 \%$ mortality (Figure 1B). To determine whether immunocompromised nude mice were particularly sensitive to the combination, we treated immunocompetent C57BL/6 mice with the metformin $+\mathrm{HCQ}$, which resulted in $30 \%$ lethality.

We then sought to determine the basis for the toxicity of metformin+HCQ combination in autopsy studies and found that body weights were not significantly different (Table 1) among the different groups. While organ weights were not different among the groups, we observed via transmission electron microscopy an increase in the number of autophagosomes in the heart, liver and kidneys of athymic nude mice treated with metformin+HCQ combination (Figure 1C). While the hematological findings were not different among the groups, we found that lactate dehydrogenase (LDH) and creatine kinase (CK) levels were elevated in all treatment groups as compared to control vehicle treated group (Table 1).

Discussion. Between March 16 and 21, 2020, partly based on a non-randomized study using HCQ with azithromycin [4] , claims disseminated through Twitter and amplified by the media that CQ or HCQ could be a therapy for COVID-19. Many individuals have started to take this drug, resulting in chloroquine poisoning in Nigeria (@NCDCgov \#COVID19Nigeria) and a death in Arizona. Notably, CQ and HCQ doses which are used for the treatment of rheumatic diseases, could lead to the development of hypoglycemia, cardiomyopathy and retinopathy [5]. Here we report the lethality of metformin $+\mathrm{CQ}$ or $+\mathrm{HCQ}$ as a warning of its potential deadly toxicity, noting that the dosages in mice are similar to those in human with allometric scaling. Consistent with our findings, the combination of $\mathrm{CQ}$ and metformin resulted in CNS neuronal damage after cardiac arrest in rats [6]. We hope that our report will be helpful to stimulate 
pharmacovigilance and monitoring of adverse drug reactions with the use of CQ or $\mathrm{HCQ}$, particularly in combination with metformin.

\section{Author contributions:}

Concept and design: Rajeshkumar, Maitra, Hidalgo, Dang

Acquisition, analysis or interpretation of data: Rajeshkumar, Yabuuchi, Pai, Dang, Hidalgo

Drafting of manuscript: Dang, Hidalgo, Rajeshkumar

Statistical analysis: Rajeshkumar, Yabuuchi

Obtained funding: Dang, Maitra, Hidalgo, Rajeshkumar

Drs. Dang and Hidalgo have access to primary data in Dr. Rajeshkumar's possession.

Conflict of Interest Disclosures: $\mathrm{MH}$, Stock and Ownership Interests: Champions Oncology, Pharmacyte Biotech, BioOncotech, Nelum, Agenus; Honoraria: Agenus, Erytech Pharma, Pharmacyte Biotech, Oncomatryx Biopharma S.L., IndMex, BioOncotech, Takeda; Consulting or Advisory Role: Oncomatryx Biopharma S.L., Takeda, Pharmacyte, Agenus, InxMed, BioOncotech; Research Funding: BiolineRx, Erytech Pharma; Patents, Royalties, Other Intellectual Property: Myriad Genetics. CVD, Stock: Agios Pharma; Board of Director member: Rafael Pharm; Consultant or Advisory: Dracen Pharm, Polaris Pharm, Geneos Pharm, Barer Institute. AM, Royalties: Cosmos Wisdom Biotechnology; License: Thrive Earlier Detection.

Funding/Support. This study was partially supported by funding from a Stand Up To Cancer Dream Team Translational Cancer Research grant (grant number: SU2C-AACR- DT0509; to N.V. Rajeshkumar, M. Hidalgo, A. Maitra and C.V. Dang). Dang is support by the Ludwig Institute for Cancer Research.

Role of the Funder/Sponsor: The funders had no role in the design and conduct of the study; collection, management, analysis, and interpretation of the data; preparation, review, or approval of the manuscript; and decision to submit the manuscript for publication. 


\section{REFERENCES}

1. Cortegiani A, Ingoglia G, Ippolito M, Giarratano A, Einav S. A systematic review on the efficacy and safety of chloroquine for the treatment of COVID-19. J Crit Care 2020 doi: 10.1016/j.jcrc.2020.03.005.

2. Rajeshkumar NV, Yabuuchi S, Pai SG, et al. Treatment of Pancreatic Cancer Patient-Derived Xenograft Panel with Metabolic Inhibitors Reveals Efficacy of Phenformin. Clin Cancer Res 2017;23(18):5639-47 doi: 10.1158/1078-0432.CCR-17-1115.

3. Yang A, Rajeshkumar NV, Wang X, et al. Autophagy is critical for pancreatic tumor growth and progression in tumors with p53 alterations. Cancer Discov 2014;4(8):905-13 doi: 10.1158/2159-8290.CD-14-0362.

4. Gautret $P$, Lagier JC, Parola $P$, et al. Hydroxychloroquine and azithromycin as a treatment of COVID-19: results of an open-label non-randomized clinical trial. Int J Antimicrob Agents 2020:105949 doi: 10.1016/j.jjantimicag.2020.105949.

5. Schrezenmeier E, Dorner T. Mechanisms of action of hydroxychloroquine and chloroquine: implications for rheumatology. Nat Rev Rheumatol 2020;16(3):155-66 doi: 10.1038/s41584-020-0372-x.

6. Zhu J, Liu K, Huang K, et al. Metformin Improves Neurologic Outcome Via AMP-Activated Protein Kinase-Mediated Autophagy Activation in a Rat Model of Cardiac Arrest and Resuscitation. J Am Heart Assoc 2018;7(12) doi: 10.1161/JAHA.117.008389. 
Figure $1 \mathrm{~A}$

B

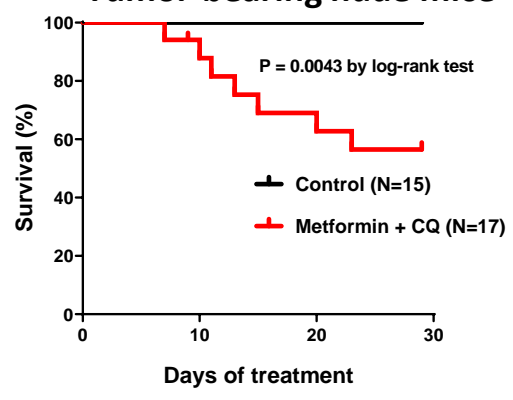

Non-tumor-bearing nude mice

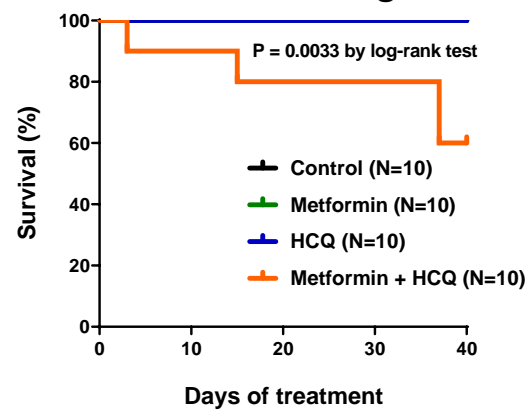

Heart

C

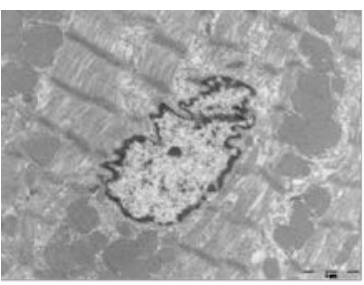

Control

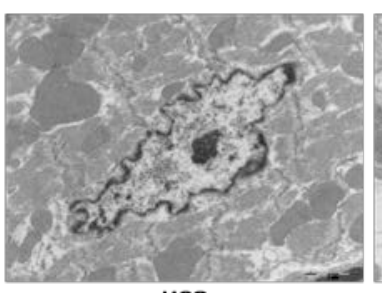

HCQ

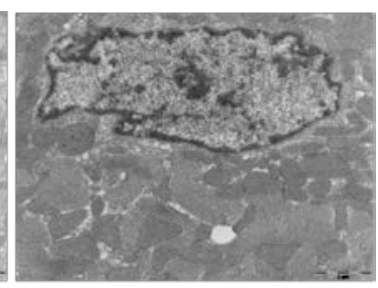

Metformin

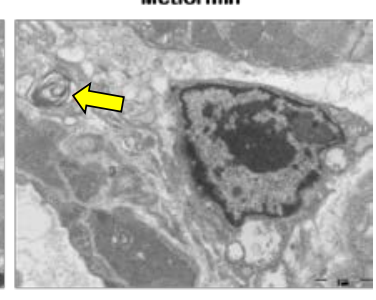

Metformin + HCQ
Non-tumor-bearing nude mice

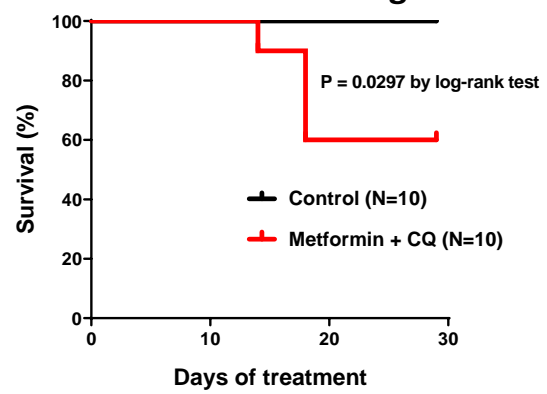

Non-tumor-bearing C57BL/6 mice

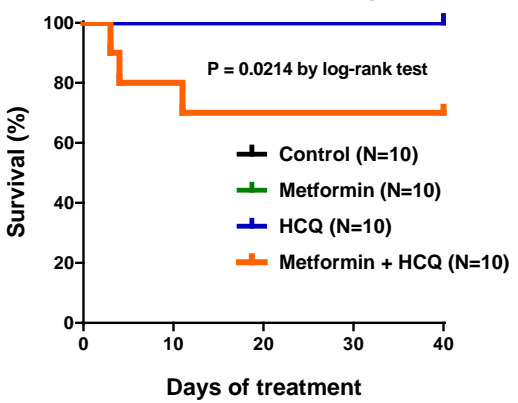

Liver

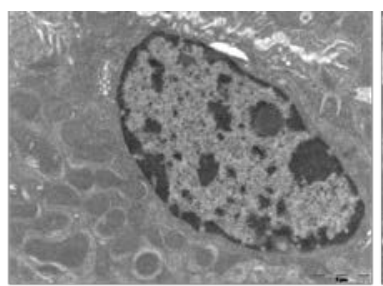

Control

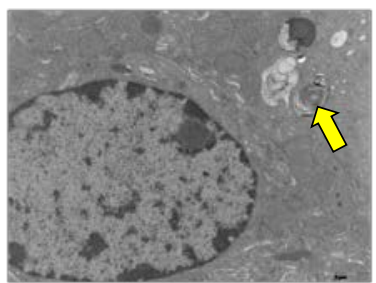

HCQ

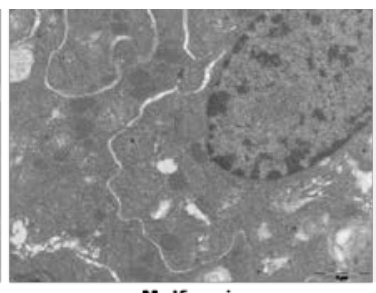

Metformin

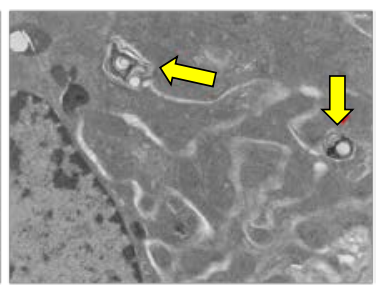

Metformin + HCQ

\section{Kidney}
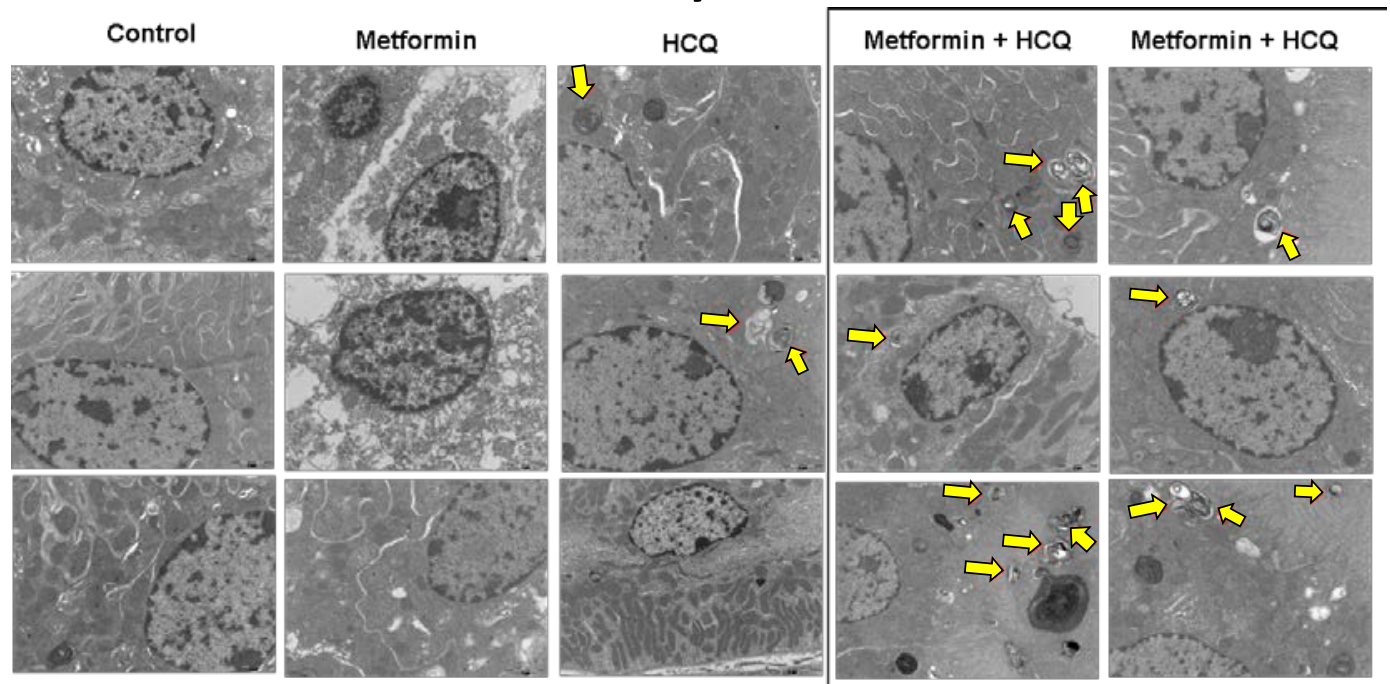
Table 1. Body weight, organ weight, $C B C$ and clinical chemistry parameters of mice administered with Vehicle (Control), Metformin, HCQ or Metformin and HCQ.

\begin{tabular}{|c|c|c|c|c|c|c|c|c|c|c|c|c|c|c|c|c|c|}
\hline \multirow{3}{*}{ Parameter } & \multirow{3}{*}{ Unit } & \multicolumn{8}{|c|}{ Non-tumor-bearing nude mice } & \multicolumn{8}{|c|}{ Non-tumor-bearing C57B/L6 mice } \\
\hline & & \multicolumn{2}{|c|}{ Control } & \multicolumn{2}{|c|}{ Metformin } & \multicolumn{2}{|c|}{ HCQ } & \multicolumn{2}{|c|}{ Metformin+HCQ } & \multicolumn{2}{|c|}{ Control } & \multicolumn{2}{|c|}{ Metformin } & \multicolumn{2}{|c|}{ HCQ } & \multicolumn{2}{|c|}{ Metformin+HCQ } \\
\hline & & Average & STDEV & Average & STDEV & Average & STDEV & Average & STDEV & Average & STDEV & Average & STDEV & Average & STDEV & Average & STDEV \\
\hline Body weight & g & 27.585 & 2.149 & 29.697 & 1.043 & 28.973 & 3.166 & 29.301 & 0.194 & 29.240 & 0.793 & 28.109 & 0.564 & 28.064 & 3.339 & 27.774 & 2.169 \\
\hline \multirow{2}{*}{\multicolumn{18}{|c|}{\begin{tabular}{|l|} 
Organ weight \\
\end{tabular}}} \\
\hline & & & & & & & & & & & & & & & & & \\
\hline \begin{tabular}{|l|l} 
Liver \\
\end{tabular} & $\mathrm{g}$ & 1.510 & 0.336 & 1.847 & 0.152 & 1.873 & 0.274 & 1.790 & 0.113 & 1.74 & 0.217 & 1.590 & 0.131 & 1.723 & 0.323 & 1.523 & 0.176 \\
\hline Spleen & $\mathrm{g}$ & 0.123 & 0.023 & 0.143 & 0.021 & 0.153 & 0.042 & 0.117 & 0.006 & 0.067 & 0.012 & 0.107 & 0.064 & 0.097 & 0.015 & 0.070 & 0.010 \\
\hline \begin{tabular}{|l|} 
Heart \\
\end{tabular} & $\mathrm{g}$ & 0.220 & 0.035 & 0.223 & 0.038 & 0.167 & 0.040 & 0.173 & 0.006 & 0.173 & 0.015 & 0.183 & 0.006 & 0.167 & 0.032 & 0.187 & 0.012 \\
\hline R Kidney & $\mathrm{g}$ & 0.297 & 0.051 & 0.360 & 0.026 & 0.360 & 0.020 & 0.303 & 0.032 & 0.293 & 0.015 & 0.297 & 0.012 & 0.290 & 0.046 & 0.277 & 0.029 \\
\hline \multirow[t]{2}{*}{ L Kidney } & $\mathrm{g}$ & 0.307 & 0.023 & 0.353 & 0.031 & 0.343 & 0.046 & 0.307 & 0.031 & 0.250 & 0.01 & 0.263 & 0.031 & 0.260 & 0.035 & 0.257 & 0.015 \\
\hline \multirow{2}{*}{\multicolumn{18}{|c|}{ CBC }} \\
\hline & & & & & & & & & & & & & & & & & \\
\hline WBC & K/uL & 3.10 & 0.399 & 3.75 & 1.194 & 10.21 & 1.785 & 11.13 & 4.427 & 6.36 & 1.048 & 7.57 & 4.603 & 5.01 & 0.752 & 4.35 & 1.314 \\
\hline Neut & $\mathrm{K} / \mathrm{uL}$ & 1.43 & 0.483 & 1.19 & 0.433 & 4.37 & 0.709 & 3.57 & 0.548 & 1.77 & 1.039 & 2.88 & 3.590 & 1.29 & 0.168 & 1.04 & 0.477 \\
\hline Lymph & K/uL & 1.57 & 0.137 & 2.27 & 0.585 & 3.68 & 1.325 & 6.28 & 3.907 & 4.15 & 0.497 & 3.95 & 0.196 & 3.52 & 0.633 & 3.00 & 0.775 \\
\hline Mono & K/uL & 0.08 & 0.040 & 0.12 & 0.090 & 0.51 & 0.098 & 0.39 & 0.245 & 0.39 & 0.177 & 0.52 & 0.610 & 0.16 & 0.025 & 0.20 & 0.133 \\
\hline RBC & $M / U L$ & 9.81 & 0.419 & 9.42 & 0.104 & 9.02 & 0.525 & 8.83 & 0.285 & 9.09 & 0.199 & 8.95 & 0.915 & 9.18 & 0.184 & 10.11 & 0.492 \\
\hline $\mathrm{Hb}$ & $\mathrm{g} / \mathrm{dL}$ & 14.80 & 1.054 & 13.67 & 0.651 & 13.20 & 0.889 & 13.03 & 0.702 & 12.60 & 0.520 & 11.97 & 1.041 & 12.07 & 0.115 & 13.37 & 0.473 \\
\hline Hct & $\%$ & 53.27 & 2.831 & 49.87 & 3.721 & 45.87 & 3.444 & 47.87 & 3.870 & 40.83 & 1.250 & 40.53 & 4.557 & 40.80 & 0.60 & 45.93 & 1.721 \\
\hline PLT & $\mathrm{K} / \mathrm{uL}$ & 1058.33 & 73.433 & 711.33 & 298.574 & 496.00 & 376.427 & 1094.67 & 188.988 & 1170.67 & 256.995 & 1206.67 & 86.524 & 1241.33 & 48.211 & 1183.33 & 120.101 \\
\hline \multicolumn{18}{|c|}{ Clinical Chemistry } \\
\hline \begin{tabular}{|l|} 
Chol \\
\end{tabular} & $\mathrm{mg} / \mathrm{dL}$ & 140.33 & 13.577 & 173.00 & 2.646 & 150.00 & 4.359 & 131.33 & 10.693 & 128.00 & 3.00 & 114.33 & 5.033 & 112.33 & 9.074 & 120.33 & 7.234 \\
\hline Trig & $\mathrm{mg} / \mathrm{dL}$ & 108.00 & 17.578 & 103.67 & 13.503 & 109.00 & 30.790 & 169.33 & 90.473 & 97.33 & 5.508 & 113.67 & 30.746 & 97.33 & 18.610 & 93.00 & 31.241 \\
\hline UA & $\mathrm{mg} / \mathrm{dL}$ & 3.00 & 0.794 & 3.40 & 1.386 & 4.13 & 0.839 & 6.47 & 3.190 & 2.90 & 0.346 & 3.63 & 0.321 & 4.83 & 0.404 & 4.03 & 0.808 \\
\hline CK & $U / L$ & 206.33 & 78.806 & 406.33 & 140.920 & 1084.00 & 454.175 & \begin{tabular}{|l}
452.67 \\
\end{tabular} & 164.214 & 240 & 138.098 & 215.00 & 102.504 & 503.67 & 349.632 & 205.67 & 97.828 \\
\hline \begin{tabular}{|l|}
$\mathrm{g}$-GTP \\
\end{tabular} & $U / L$ & 3.00 & 1.732 & 3.67 & \begin{tabular}{|l|}
0.577 \\
\end{tabular} & 3.00 & 1.00 & 3.00 & $\begin{array}{l}1.732 \\
\end{array}$ & \begin{tabular}{|l|}
1.67 \\
\end{tabular} & 2.082 & 3.00 & 1.000 & 3.33 & 1.155 & 3.33 & 1.155 \\
\hline \begin{tabular}{|l|} 
ALT \\
\end{tabular} & $U / L$ & 59.33 & 19.088 & 58.33 & 6.658 & 52.00 & 5.568 & 100.67 & 68.061 & 54.67 & 5.033 & 82.00 & 35.791 & 67.67 & 11.930 & 71.67 & \begin{tabular}{|l|}
6.028 \\
\end{tabular} \\
\hline \begin{tabular}{|l|} 
AST \\
\end{tabular} & $U / L$ & 103.33 & 21.385 & 163.00 & 17.349 & 219.67 & 4.163 & 233.67 & 122.786 & \begin{tabular}{|l|}
92.67 \\
\end{tabular} & 23.159 & 187.33 & 79.387 & 168.00 & 32.419 & 145.33 & 9.504 \\
\hline Amyl & $U / L$ & 969.67 & 261.077 & 1205.00 & 122.135 & 1192.00 & 196.929 & 1313.33 & 129.732 & 1264.00 & 204.648 & 1607.33 & 383.981 & 1546.33 & 260.064 & 1563.00 & 78.848 \\
\hline \begin{tabular}{|l|} 
ALP \\
\end{tabular} & $U / L$ & 112.00 & 11.000 & 121.67 & 10.599 & 96.33 & 11.015 & 74.67 & 8.737 & 75.33 & 3.055 & 90.67 & 31.974 & 84.33 & 3.512 & 87.67 & 16.862 \\
\hline T-Bil & $\mathrm{mg} / \mathrm{dL}$ & $\begin{array}{l}0.20 \\
\end{array}$ & 0.000 & 0.23 & \begin{tabular}{|l|l|}
0.058 \\
\end{tabular} & \begin{tabular}{|l|}
0.27 \\
\end{tabular} & 0.058 & 0.30 & $\begin{array}{l} \\
\end{array}$ & 0.43 & 0.058 & \begin{tabular}{|l|}
0.27 \\
\end{tabular} & 0.058 & 0.23 & 0.058 & 0.23 & 0.058 \\
\hline $\mid$\begin{tabular}{|l|} 
Direct-Bil \\
\end{tabular} & $\mathrm{mg} / \mathrm{dL}$ & 0.10 & 0.000 & 0.17 & 0.058 & 0.27 & 0.115 & 0.20 & 0.100 & 0.47 & 0.058 & 0.27 & 0.058 & 0.17 & 0.058 & 0.20 & 0.100 \\
\hline \begin{tabular}{|l|} 
Glu \\
\end{tabular} & $\mathrm{mg} / \mathrm{dL}$ & 227.67 & 44.970 & 225.67 & 31.943 & 257.00 & 40.951 & 356.33 & 117.619 & 237.67 & 42.028 & 192.67 & 75.936 & 244.00 & 23.065 & 261.00 & 64.257 \\
\hline \begin{tabular}{|l|} 
T-protein \\
\end{tabular} & $\mathrm{g} / \mathrm{dL}$ & 5.20 & 0.173 & 5.47 & 0.231 & 5.40 & 0.173 & 4.50 & 0.781 & 6.00 & 0.10 & 5.63 & 0.153 & 5.87 & 0.058 & 5.63 & 0.252 \\
\hline $\begin{array}{ll}\mathrm{Ca} \\
\end{array}$ & $\mathrm{mg} / \mathrm{dL}$ & 10.73 & 0.153 & 10.77 & 0.643 & 11.17 & 0.306 & 11.73 & 1.686 & 10.10 & 0.436 & 10.17 & 0.451 & 10.63 & 0.231 & 10.20 & 0.100 \\
\hline BUN & $\mathrm{mg} / \mathrm{dL}$ & 20.67 & 1.528 & 22.33 & 3.055 & \begin{tabular}{|l|}
19.00 \\
\end{tabular} & 5.292 & 17.33 & 3.786 & \begin{tabular}{|l|}
19.00 \\
\end{tabular} & 1.732 & \begin{tabular}{|l|}
15.67 \\
\end{tabular} & 4.726 & 14.00 & 3.606 & 13.33 & 1.528 \\
\hline \begin{tabular}{|l|} 
Cre \\
\end{tabular} & $\mathrm{mg} / \mathrm{dL}$ & 0.40 & 0.000 & 0.37 & 0.058 & 0.33 & 0.058 & 0.33 & 0.058 & 0.20 & 0.2 & 0.30 & 0.000 & 0.43 & 0.058 & 0.40 & 0.000 \\
\hline Albumin & $U / L$ & 3.17 & 0.153 & 3.20 & 0.10 & 3.13 & 0.115 & 2.90 & 0.00 & 3.47 & 0.153 & 3.07 & 0.493 & 3.17 & 0.058 & 3.17 & 0.058 \\
\hline LDH & $U / L$ & 309.00 & 39.611 & 3077.67 & 343.08 & 2665.67 & 319.639 & 2645.33 & 392.263 & 478.33 & 54.372 & 2884.00 & $\mid 1018.500$ & 2879.67 & 533.209 & 2376.00 & 337.311 \\
\hline HDL & $\mathrm{mg} / \mathrm{dL}$ & 82.67 & 8.622 & 97.33 & 3.51 & 84.33 & 4.041 & 80.33 & 3.786 & 78.33 & 3.055 & 65.00 & 10.440 & 67.00 & 6.245 & 68.67 & 1.528 \\
\hline
\end{tabular}

